\title{
Physical fitness and psycho-cognitive performance in the young and middle-aged workforce with primarily physical versus mental work demands
}

\author{
Olaf Prieske $^{1}$ D $\cdot$ Tina Dalager $^{2} \cdot$ Vanessa Looks $^{1} \cdot$ Kathleen Golle $^{1} \cdot$ Urs Granacher $^{1}$
}

Received: 27 March 2019 / Accepted: 4 June 2019 / Published online: 26 June 2019

(C) The Author(s) 2019

\begin{abstract}
Aim The purpose of this study was to examine physical fitness and psycho-cognitive performance and their associations in young and middle-aged workers with primarily physical versus mental work demands.

Subjects and methods Healthy young and middle-aged workers ( 73 men, age $=33 \pm 7$ years; 75 women, age $=35 \pm 9$ years) were recruited from German small-to-medium-sized enterprises ( $<250$ employees) and classified into groups with primarily mental (MD) or physical demands (PD) at work. Participants were tested for cardiorespiratory fitness, trunk flexor/extensor muscular endurance, handgrip strength, balance, leg muscle power, perceived stress, cognitive performance, and work ability. Results Ninety-four workers were allocated to the MD (53\% females) and 54 to the PD (46\% females) groups. The MD group showed significantly better balance, trunk extensor muscular endurance, and cognitive performance $(p<0.035,0.35 \leq d \leq 0.55)$ and less stress compared with the PD group $(p<0.023, d=0.38)$. Group-specific Spearman rank correlation analysis $\left(r_{\mathrm{S}}\right)$ revealed significant small-to-medium-sized correlations between physical fitness and cognitive performance $\left(-0.205 \leq r_{\mathrm{S}} \leq\right.$ $0.434)$ in the MD and PD groups. Significant small-to-medium-sized correlations were found for physical fitness and stress/work ability $\left(0.211 \leq r_{\mathrm{S}} \leq 0.301\right)$ in the MD group only. Further, associations of trunk extensor muscular endurance and work ability were significantly higher in the MD group $\left(r_{\mathrm{S}}=0.240\right)$ compared with the PD group $\left(r_{\mathrm{S}}=-0.141 ; \mathrm{z}=2.16, p=0.031\right)$.

Conclusions MD workers showed better physical fitness measures (balance, trunk extensor muscular endurance) and cognitive performance and lower levels of perceived stress compared with PD workers. Small-to-medium-sized associations between physical fitness and psycho-cognitive performance measures indicate that gains in physical fitness may at least partly contribute to psycho-cognitive performance and/or vice versa, particularly in MD workers.
\end{abstract}

Keywords Core strength $\cdot$ Endurance $\cdot$ Stress $\cdot$ Work ability $\cdot$ Association

\section{Introduction}

Physical fitness is an important marker of health in adults (Bouchard et al. 2012; Sjøgaard et al. 2016). For instance, higher levels of physical fitness as indicated by upper- and lower-body strength are associated with a lower risk of all-

Olaf Prieske

prieske@uni-potsdam.de

1 Division of Training and Movement Sciences, Research Focus Cognitive Sciences, University of Potsdam, Am Neuen Palais 10, 14469 Potsdam, Germany

2 Department of Sports Science and Clinical Biomechanics, University of Southern Denmark, Odense, Denmark cause mortality in adults, irrespective of age (García-Hermoso et al. 2018). Further, gains in physical fitness (i.e., balance, lower limb muscle strength) following 8 weeks of combined balance and strength training improved gait performance (i.e., habitual gait speed) in middle-aged workers (Granacher et al. 2011b). Additionally, components of physical fitness are related to psycho-cognitive performances (e.g., short-term memory, stress resilience) (Cassilhas et al. 2007; LiuAmbrose et al. 2010; Forcier et al. 2006; Tonello et al. 2014). A meta-analysis examined associations between measures of cardiorespiratory fitness (CRF) and cardiovascular reactivity to psychological stressors in adults (Forcier et al. 2006). Individuals with higher levels of CRF revealed lower values of heart rate reactivity as a physiological measure of acute psychological stress (Forcier et al. 2006). Moreover, a longitudinal study examined the effects of a 24 -week 
resistance training program on cognitive performances (e.g., short-term memory, attention) in sedentary old adults and found significant gains in maximum strength (e.g., chest press, leg press), together with improved short-term and long-term memory, as well as attention (Cassilhas et al. 2007). Consequently, gains in physical fitness can be translated to improvements in psycho-cognitive performances.

In the working population, physical fitness as well as psycho-cognitive performances can be affected by physical demands at work (Holtermann et al. 2010; Savinainen et al. 2004; Torgén et al. 1999; Then et al. 2014). More specifically, physical work demands such as peak loadings, repetitive and fatiguing movements, or constrained postures may induce muscle pain and compromise musculoskeletal function, as well as mental health (Sjøgaard et al. 2016; Søgaard and Sjøgaard 2017). In fact, Savinainen et al. (2004) examined the effects of physical work demands as indicated by ratings of perceived exertion (RPE, scale 6-20) during a working day. Among middle-aged Finnish municipal workers, components of physical fitness (e.g., muscle strength, CRF) were associated with higher levels of muscle strength (e.g., maximum trunk extensor and handgrip strength) in workers with low (RPE $\leq$ 12.8 and 12.5 in males and females, respectively) compared with high physical workloads (RPE $>12.8$ and 12.5 in males and females, respectively). Further, better outcomes of cognitive performance (e.g., short-term memory, semantic memory) were observed in middle-aged and old workers with high versus low mental work demands (Then et al. 2014). Moreover, middle-aged workers in less physically demanding jobs (i.e., white-collar workers) experienced more mental stress at the worksite compared with workers who experienced high physical work demands (i.e., blue-collar workers) (Wu and Porell 2000). Thus, workers with primarily mental work demands can have higher levels of physical fitness (e.g., muscle strength), cognitive performance (e.g., short-term memory), and perceived psychological stress compared with workers with physical work demands.

With respect to higher levels of physical fitness, cognitive performance, and psychological stress in mental compared to physical work demands, associations between measures of physical fitness and psycho-cognitive performance may be more pronounced in workers with mental work demands. However, it is unresolved if measures of physical fitness (e.g., CRF, muscle strength, balance) and psycho-cognitive performances (i.e., short-term memory, perceived stress, work ability), as well as their relationships, are different in young and middle-aged workers with physical versus mental work demands. A better understanding on physical and psychocognitive performance measures in workers with primarily physical versus mental work demands may help to promote exercise training programs for employees with specific work demands (Søgaard and Sjøgaard 2017). The purpose of this study was to examine measures of physical fitness and psycho-cognitive performances in the young and middleaged workforce with primarily physical versus mental work demands. In addition, we aimed to assess the associations between the measures of physical fitness and psychocognitive performances. Based on the relevant literature (Savinainen et al. 2004; Søgaard and Sjøgaard 2017; Then et al. 2014; Wu and Porell 2000), we hypothesized greater fitness measures and better cognitive performances together with higher levels of perceived stress in workers with primarily mental compared with physical work demands. Further, we expected significant positive associations between variables of physical fitness and cognitive performances and significant negative associations between physical fitness and perceived stress (Forcier et al. 2006; Cassilhas et al. 2007), particularly in workers with primarily mental work demands.

\section{Methods}

\section{Participants}

Participant recruitment occurred from September 2015 to March 2016 during a public health promotion campaign by a German health insurance company. Small-to-medium-sized companies $(<250$ employees) in the north-east of Germany were contacted by the insurance company and asked to participate in the health promotion campaign. All companies were members of a public health promotion network of the insurance company comprising, overall, 224 companies and $\sim 55,700$ employees. To be eligible for inclusion in this study, participants had to be healthy according to the Physical Activity Readiness Questionnaire (Jamnik et al. 2007) and aged between 18 and 49 years (Granacher et al. 2011b). Participants were excluded if they reported acute/chronic pain, injuries within the last 6 months that needed medical treatment, and/or any type of musculoskeletal or neurological disorders that may have affected the outcomes of this study (e.g., arthritis, multiple sclerosis). With reference to the study of Savinainen et al. (2004), an a priori power analysis with an assumed Type I error rate of 0.05 and $80 \%$ statistical power was computed (Faul et al. 2009). The analysis indicated that 128 workers are sufficient to observe a medium-sized main effect of group (physical versus mental work demand) on handgrip strength as a global fitness measure. Eighty-seven males and 86 females from eight companies volunteered to participate. Due to poor health conditions indicated by the Physical Activity Readiness Questionnaire, 14 males and 11 females were excluded. Thus, 73 healthy male (mean age $32.8 \pm 7.4$ years) and 75 healthy female workers (mean age $35.0 \pm 9.0$ years) were finally included in this study. The number of participants per company ranged from 8 to 33 . Participants were working for two food companies $(n=61)$, an insurance company $(n=14)$, civil service $(n=13)$, social 
service $(n=10)$, a crafts producer $(n=19)$, hotel business $(n=$ $23)$, and a healthcare business $(n=8)$.

\section{Design and experimental procedure}

For the present study, a cross-sectional study design was used. As part of a public health promotion campaign, participants were tested at the worksite in an undisturbed room provided by the respective company. The campaign was organized by the health insurance company and the main focus was to determine the employees' physical fitness and psycho-cognitive performances.

\section{Material and instruments}

Cognitive performances, perceived stress, and work ability were assessed by standardized questionnaires. Physical tests comprised the assessment of CRF, handgrip strength, trunk muscle endurance, jump performance, and balance. In addition, workers' leisure-time physical activity (LTPA) was assessed using the Freiburg questionnaire for everyday and sports-related activities (Frey et al. 1999). Because the main focus of the campaign was on the employees' physical fitness and psycho-cognitive performances, factors such as lifestyle, socioeconomic status, and/or education were not examined here. Tests were always conducted by the same experienced exercise scientists and/or physiotherapists. Testing started with the assessment of cognitive performance and balance in order to avoid fatigue-related effects on performance output. All other tests were conducted in a randomized order.

Cardiorespiratory fitness was determined by means of a modified Harvard step test (Ryhming 1953). The exercise test consisted of stepping up and down from a platform at a rate of 120 steps per minute for $3 \mathrm{~min}$. Participants' heart rate was assessed at rest before the test $\left(\mathrm{HR}_{1}\right)$, immediately after the test $\left(\mathrm{HR}_{2}\right)$, and at $1 \mathrm{~min}$ recovery after the test $\left(\mathrm{HR}_{3}\right)$ using a wearable heart rate monitor (RCX5, Polar Electro $\mathrm{GmbH}$, Buettelborn, Germany). The CRF index was calculated using the following formula of Ruffier: $\mathrm{CRF}$ index $=\left(\mathrm{HR}_{1}+\mathrm{HR}_{2}+\right.$ $\left.\mathrm{HR}_{3}-200\right) / 10$ (Nsenga Leunkeu et al. 2014).

Handgrip strength of the dominant hand was assessed using a hand-held dynamometer. During testing, participants were standing in an upright erect position with both arms straight at the side and the dynamometer in the dominant hand (Suni et al. 2014). Participants were instructed to press the dynamometer grip as forcefully as possible for $5 \mathrm{~s}$ while maintaining the upright position (i.e., no additional movements from upper or lower body). Two trials with a 30-s rest between trials were conducted and the best trial in terms of maximal strength was used for further analysis.

Muscular endurance of the trunk flexors was assessed with a dynamic plank test as described by Bourban et al. (2001). During testing, participants were in prone bridge position on their elbows and toes. Participants were asked to lift their feet alternately for 2 to $5 \mathrm{~cm}$ according to the beat of a metronome (i.e., 60 beats per minute) for as long as possible. Verbal warnings were given when participants lost touch to a horizontal reference rod or failed to lift their feet to the beat. The test was terminated when participants failed to remain in contact with the reference rod for the third time. Test time until failure of one trial was manually measured by the tester using a hand-held stopwatch and was used for further analysis.

Muscular endurance of the trunk extensors was assessed by means of a modified Biering-Sørensen test (Biering-Sørensen 1984). Participants lay in prone position on a padded examination table and maintained the unsupported trunk (from superior border of the iliac crest) with arms folded across the chest in horizontal position until failure. Failure was defined as the time point when participants could no longer hold the horizontal position. The lower body was manually fixed on the examination table at the level of the shanks. Time until failure was assessed with a hand-held stopwatch and used for further analysis.

The jump-and-reach test was used as a proxy of leg muscle power (Suni et al. 2014). Before the test started, standing reach height was assessed during upright erect standing with both feet in lateral position close to a wall and the dominant arm fully extended in upright direction and the fingers touched a scale at the wall. Subsequently, participants performed a countermovement (i.e., knee and hip flexion) with arm swing immediately followed by a rapid and powerful vertical jump. The participants' task was to touch the wall-mounted scale during flight time at the highest position with their middle finger. Two familiarization and two test trials were allowed. The difference between standing reach height and jumping reach height was defined as jump height and the best trial was used for analysis.

Static balance was tested for $30 \mathrm{~s}$ during single-leg stance (Bohannon et al. 1984). The test involved participants to stand barefoot on their dominant leg (Coren 1993) with hands akimbo and eyes closed. Leg dominance was assessed using the lateral preference. The test was terminated when hands were lifted from the hips, eyes were opened, the free leg touched the floor or the supporting leg moved from the initial standing position. A second test trial was conducted if the first trial was $<30 \mathrm{~s}$. The best trial in terms of standing time was used for further analysis.

Cognitive performance was assessed with the digit symbol substitution test as a measure of the participants' attention and short-term memory (Lezak et al. 2012). In brief, the digit symbol substitution test requires transcribing geometric symbols to Arabic digits according to a specific coding table. Following a familiarization trial, participants were asked to copy as many corresponding symbols to a list of digits within $90 \mathrm{~s}$. The number of correct symbols was used for analysis. Higher test values indicate better cognitive performance. 
Level of perceived stress was quantified using the tenitem Perceived Stress Scale (Cohen et al. 1983). Participants responded on a five-point scale ranging from 0 (never) to 4 (very often) to identify how unpredictable, uncontrollable, and overloading participants estimated their lives. Lower stress scores represent lower levels of perceives stress.

Work ability was measured using the work ability index (WAI) questionnaire. WAI is a sum variable based on the subjective estimation of the ability to work in relation to physical and mental work demands and mental resources (Ilmarinen 2009). The WAI questionnaire deals with seven items (e.g., physical and mental work demands, diagnosed diseases, occurrence of sick leave). The WAI sum score can range from 7 to 49 points, with higher values indicating larger work ability. The sum WAI score was used for further analysis. Additionally, based on the first question of the questionnaire (i.e., subjective evaluation of the work demands in relation to both physical and mental demands), participants were classified as workers with "primarily physical demands at work" (PD group) or "primarily mental demands at work" (MD group).

\section{Statistical analyses}

The test of work ability (i.e., question on work demands) was used to categorize participants as workers with primarily "physical demands at work" (PD group) or workers with primarily "mental demands at work" (MD group). Descriptive data are presented as group mean values and standard deviations, as well as median and interquartile range (25th to 75 th percentile), respectively. Normal distribution was examined using the Kolmogorov-Smirnov test. Due to the violation of normal distribution in most of our outcome measures, the nonparametric Mann-Whitney U-test for independent samples was used to compute group-specific effects (i.e., PD vs. $\mathrm{MD}$ ) for measures of physical fitness and psycho-cognitive performances. Subgroup analyses were performed for LTPA using the median split approach based on the whole group (i.e., low LTPA $<7.1 \mathrm{~h} /$ week, average intensity: metabolic equivalent $[\mathrm{MET}] / \mathrm{h}=4.9 \pm 0.7$, total $\mathrm{MET} /$ week $=18.6 \pm$ 11.1; high LTPA $\geq 7.1 \mathrm{~h} /$ week, average intensity: $\mathrm{MET} / \mathrm{h}=$ $4.8 \pm 0.8$, total $\mathrm{MET} /$ week $=66.6 \pm 32.0)$ and the nonparametric Kruskal-Wallis test for independent samples (i.e., PD with low LTPA vs. PD with high LTPA vs. MD with low LTPA vs. MD with high LTPA) (Moore et al. 2013). Post-hoc tests for the subgroup analyses were conducted using the Bonferroni-adjusted $\alpha$ to identify the comparisons that were statistically significant. The significance level was set at $p<0.05$. Effect sizes were determined using the formula $r_{R}$ $=|Z / \sqrt{N}|$ and converting $r_{\mathrm{R}}$ to Cohen's $d$. According to Cohen (1992), effect sizes can be classified as small $(d<$ $0.5)$, medium $(0.5 \leq d<0.8)$, and large $(d \geq 0.8)$.
Additionally, a non-parametric rank correlation coefficient (Spearman's Rho $[\rho]$ ) was calculated to assess relationships between measures of physical fitness and psycho-cognitive performances. Correlation coefficients can be classified as small $(\rho<0.3)$, medium $(0.3 \leq \rho<0.5)$, and large $(\rho \geq 0.5)$ (Cohen 1992). Group-specific differences were computed according to $\rho$ values. For this purpose, the following formula was used: $z=\left(\rho_{z 1}-\rho_{z 2}\right) / \sqrt{ }\left(1 /\left(n_{1}-3\right)+1 /\left(n_{2}-3\right)\right)$. All analyses were performed using the Statistical Package for the Social Sciences (SPSS) version 25.0.

Data availability The datasets used and/or analyzed during this study are available from the corresponding author on reasonable request.

\section{Results}

Ninety-four workers were allocated to the MD group and 54 workers to the PD group. The demographic characteristics of the participants are presented in Table 1. Significant betweengroup differences were found for age and LTPA, with higher values in the MD compared with the PD group $(p<0.05)$. Subgroup analyses for LTPA revealed mean LTPA levels of $2.6 \pm 1.7 \mathrm{~h} /$ week for PD with low LTPA, $14.5 \pm 7.7 \mathrm{~h} /$ week for PD with high LTPA, $3.8 \pm 2.3 \mathrm{~h} /$ week for MD with low LTPA, and $13.7 \pm 6.6 \mathrm{~h} /$ week for MD with high LTPA.

\section{Work demands and physical fitness}

Small-sized main effects of group were found for muscular endurance of the trunk extensors $(p<0.05, d=0.37)$ and for static balance $(p<0.05, d=0.38)$. Better performances were found in the MD group compared with the PD group. Subgroup analyses for LTPA showed significant main effects of group for muscular endurance of trunk extensors $(p<0.05$, $d=0.44)$ and static balance $(p<0.05, d=0.50)$. Post-hoc tests indicated significantly higher trunk extensor muscular endurance in MD with low LTPA compared with PD with low LTPA ( $p<0.05, d=0.87$; Fig. 1a). Further, static balance was significantly better in MD with high LTPA compared with PD with low LTPA $(p<0.05, d=0.60$; Fig. 1b).

\section{Work demands and psycho-cognitive performance}

Significant small-to-medium sized main effects of group were found for cognitive performance $(p=0.001, d=0.58)$ and perceived stress $(p<0.05, d=0.40)$. Better cognitive performance and lower levels of perceived stress were demonstrated in MD compared with PD. Subgroup analyses for LTPA showed significant main effects of group for cognitive performance $(p<0.05, d=0.49)$. Post-hoc tests indicated significantly higher cognitive performance in MD with low LTPA 
Table 1 Demographic characteristics of the included participants

\begin{tabular}{lll}
\hline & MD group $(n=94)$ & PD group $(n=54)$ \\
\hline Sex (percentage of females) & $53.2 \%$ & $46.3 \%$ \\
Age (years)* & $35.2 \pm 7.6$ & $31.7 \pm 9.1$ \\
Body height $(\mathrm{cm})$ & $174.2 \pm 10.5$ & $173.9 \pm 8.4$ \\
Body mass $(\mathrm{kg})$ & $77.1 \pm 16.2$ & $77.2 \pm 14.4$ \\
Body mass index $\left(\mathrm{kg} / \mathrm{m}^{2}\right)$ & $25.2 \pm 4.2$ & $25.4 \pm 4.1$ \\
Leisure-time physical activity (h/week)* & $9.5 \pm 7.2$ & $6.3 \pm 7.1$ \\
\hline
\end{tabular}

$M D$ workers with mental demands at work; $P D$ workers with physical demands at work $* p<0.05$

compared with PD with low LTPA ( $p<0.05, d=0.69$; Fig. 1c).

\section{Associations between physical fitness and psycho-cognitive performance}

Group-specific associations between measures of physical fitness and psycho-cognitive performances are presented in Table 2 for MD and PD. Overall, the magnitude of the correlation coefficients ranged from small to medium $(-0.279 \leq \rho \leq 0.434)$. Significant correlation coefficients were primarily found between physical fitness and psychocognitive performances in the MD group. Values ranged from $\rho=-0.279$ to $\rho=0.318(p<.05)$. The magnitudes of correlation coefficients were significantly different between the PD and MD groups for associations of jump performance and cognitive performance in favor of the PD group and for associations of trunk muscle endurance (extensors) and work ability in favor of the MD group (Table 3).

\section{Discussion}

The main findings of this research were that: (i) MD showed better performances in balance, trunk extensor muscular endurance, and cognitive function compared with PD, particularly in individuals with low LTPA levels; (ii) perceived stress was lower in MD compared with PD; (iii) small-to-medium sized associations between physical fitness and psychocognitive performance measures in the workforce were more pronounced in MD.

One important finding of our cross-sectional study revealed that work demands affected components of physical fitness (i.e., balance, trunk extensor muscular endurance) in young and middle-aged employees. In fact, trunk extensor muscular endurance and static balance were better in MD compared with PD. More specifically, trunk muscle endurance was higher in MD with low LTPA compared with PD with low LTPA. In individuals with higher levels of LTPA, no performance differences were observed between MD and PD. Our results are well in line with the findings of Savinainen et al.
(2004). These authors revealed that muscle strength (e.g., maximum trunk extensor and handgrip strength) was better in workers with low (i.e., MD) compared with high physical workloads. Accordingly, the authors concluded that high physical work demands do not necessarily translate to better physical fitness in the workforce (Savinainen et al. 2004). In contrast, it has even been postulated that high physical work demands in terms of intensities or repetitions may induce musculoskeletal dysfunction, pain, and/or adverse health events (Søgaard and Sjøgaard 2017). In this regard, it was demonstrated that PD workers have higher risks for longterm sickness absence and early mortality, particularly in men (Holtermann et al. 2012; Coenen et al. 2018). From this, it follows that, in our study, employees with pronounced physical work demands could have compromised physical fitness (i.e., trunk muscle endurance, static balance). This is of particular interest because lower levels of trunk muscle endurance and static balance are associated with an increased risk of sustaining low back pain and/or falls (Biering-Sørensen 1984; Granacher et al. 2011a, 2013). Future longitudinal studies may examine whether physical versus mental work demands adversely affect physical fitness and/or health in young and middle-aged adults. It seems as if physical activity can have positive and negative effects on performance and markers of health, depending on the setting of physical activity behavior. If it is conducted during leisure time, it has beneficial effects on musculoskeletal function and health (Sjøgaard et al. 2016; Søgaard and Sjøgaard 2017; Holtermann et al. 2010, 2012). For instance, Holtermann et al. (2012) showed that higher levels of LTPA had a positive effect on long-term sickness absence. Our subgroup analyses revealed differences between MD and PD in trunk muscle endurance for individuals with low but not high LTPA. In other words, LTPA modified the effects of physical work demands on physical fitness (i.e., trunk muscle endurance). Interestingly, higher levels of physical fitness can contribute to daily activities, occupational performance, and health (Bouchard et al. 2012; Sjøgaard et al. 2016). In terms of occupational performance, associations between changes in physical fitness (e.g., trunk flexor/extensor strength) and onthe-job performance (i.e., sickness presenteeism) were 

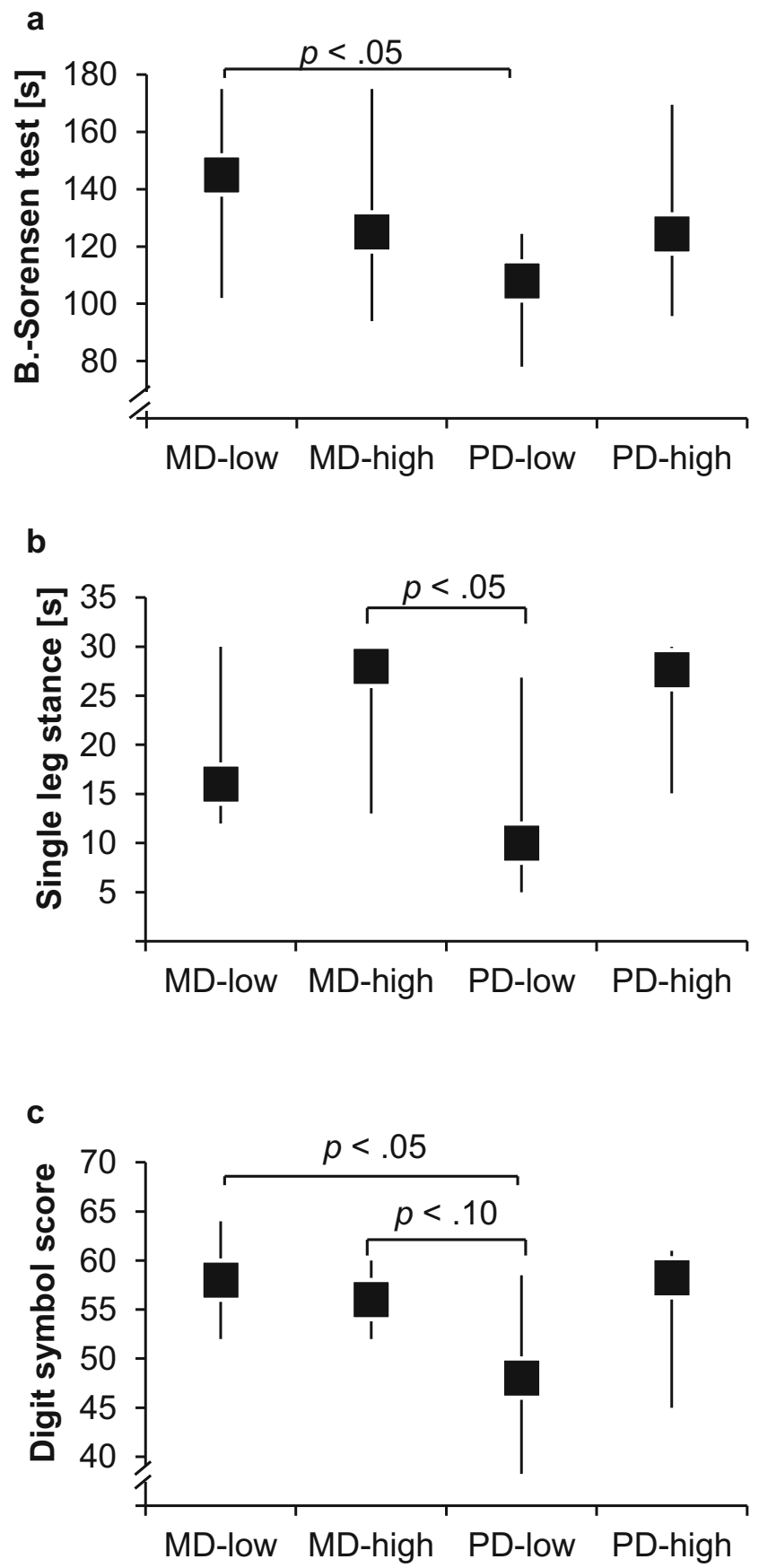

Fig. 1 Muscular endurance of the trunk extensors, static balance, and cognitive performance in workers with primarily mental (MD group) versus physical (PD group) work demands and lower (-low) versus higher (-high) levels of leisure-time physical activity. Data presented as means and $95 \%$ confidence interval

examined following 3 months of a multifactorial intervention program (e.g., strength training, cognitive behavioral training) in healthcare workers (Christensen et al. 2015). Medium-sized correlations were reported between increments in trunk flexor/ extensor strength and gains in on-the-job performance $(0.411 \leq$ Pearson's $r \leq 0.456)$, indicating the importance of physical fitness (e.g., trunk muscle strength) for occupational performance (Christensen et al. 2015).

A second finding showed that physical work demands affected cognitive performance and perceived stress in the young and middle-aged workforce. More precisely, cognitive performance was higher and perceived stress was lower in MD compared with PD. This was particularly pronounced in individuals with low LTPA levels. Recently, Then et al. (2014) examined the effects of mental work demands on cognitive performance (i.e., Trail Making Test, verbal fluency test) in adults aged 40 to 80 years. In accordance with our results, the authors reported better cognitive performances in MD compared with PD. It was suggested that particularly high mental work demands may provide optimal stimuli to improve cognitive performances (Then et al. 2014). In fact, it has previously been shown that high mental demands are essential to induce cognitive learning (Fairclough et al. 2005). In this study, MD performed better in the cognitive task (i.e., digit symbol substitution test) than PD. This might be due to the fact that $\mathrm{MD}$ experienced high mental work demands. Of note, our findings in regards of lower stress levels in MD compared with PD are in contrast with the literature. Myrtek et al. (1999) showed that white-collar workers (i.e., workers with primarily mental work demands) were more likely to experience mental stress at work compared with blue-collar workers (i.e., workers with primarily physical work demands). This discrepancy in findings can be explained by different LTPA levels in the study of Myrtek et al. (1999) as compared with our study. While we found higher LTPA levels in MD compared with PD, Myrtek et al. (1999) reported similar LTPA levels in white- and blue-collar workers. Interestingly, there is evidence that more active individuals seem to be more resilient to psychosocial stressors (Tonello et al. 2014; Teisala et al. 2014). Moreover, a meta-analysis showed that individuals with higher levels of physical fitness revealed lower levels of perceived stress (Forcier et al. 2006). We found better trunk extensor muscular endurance and static balance in MD compared with PD. Thus, it can be hypothesized that, in our study, better stress resilience in MD versus PD could be related to higher LTPA and/or fitness.

Another important finding of our study was the small-tomedium sized associations between physical fitness and psycho-cognitive performances $(-0.279 \leq \rho \leq 0.434)$, particularly in MD. These results are well in line with the literature. For instance, Teisala et al. (2014) reported medium-sized correlations between $\mathrm{CRF}\left(\mathrm{VO}_{2 \max }\right)$ and perceived stress in healthy males with a mean age of 34 years. Moreover, Newson and Kemps (2008) showed better cognitive performances (e.g., attention, working memory, processing speed) in adults (mean age 46 years) with high-level compared with low-level CRF $\left(\mathrm{VO}_{2 \max }\right)$. The findings from our cross-sectional study and the literature indicate that individuals with better fitness levels also show better psycho-cognitive performances (i.e., attention, short-term memory, perceived stress, work ability), particularly in individuals with predominantly mental work demands. 
Table 2 Measures of physical fitness and psycho-cognitive performance in workers with mental (MD) and physical (PD) demands at work

\begin{tabular}{|c|c|c|c|c|c|}
\hline & \multicolumn{2}{|c|}{ MD group } & \multicolumn{2}{|c|}{ PD group } & \multirow[b]{2}{*}{$p$-Value (Cohen's $d$ ) } \\
\hline & Median & IQR & Median & IQR & \\
\hline \multicolumn{6}{|l|}{ Physical fitness } \\
\hline CRF index & 12.6 & $8.8-15.7$ & 12.3 & $9.6-16.1$ & $0.627(0.04)$ \\
\hline Handgrip strength (kg) & 38.8 & $31.4-54.4$ & 44.9 & $35-54.6$ & $0.143(0.30)$ \\
\hline Trunk muscle endurance (flexors) (s) & 72.0 & $51.5-102.0$ & 68.0 & $44.0-96.0$ & $0.250(0.20)$ \\
\hline Trunk muscle endurance (extensors) (s) & 135.0 & $93.5-167.3$ & 110.3 & $87.5-140.8$ & $0.035(0.37)$ \\
\hline Jump performance $(\mathrm{cm})$ & 35.0 & $29.0-44.3$ & 37.5 & $30.0-44.0$ & $0.953(0.01)$ \\
\hline Static balance (s) & 21.5 & $11.8-30.0$ & 15.5 & $5.8-30.0$ & $0.029(0.38)$ \\
\hline \multicolumn{6}{|l|}{ Psycho-cognitive performance } \\
\hline Cognitive performance & 56.5 & $49.0-62.0$ & 51.9 & $43.0-58.1$ & $0.001(0.58)$ \\
\hline Perceived stress & 12 & $7.5-16.5$ & 14 & $10.5-18$ & $0.023(0.40)$ \\
\hline Work ability & 43.3 & $40.0-45.9$ & 43.0 & $39.0-44.0$ & $0.640(0.32)$ \\
\hline
\end{tabular}

$C R F$ cardiorespiratory fitness; $I Q R$ interquartile range; $M D$ workers with mental demands at work; $P D$ workers with physical demands at work

Nevertheless, future studies should examine whether physical exercise programs with the goal to improve physical fitness have the potential to induce enhancements in psychocognitive performances in MD and PD workers.

\section{Strengths and limitations of the study}

A strength of this study is that it is the first to examine physical fitness and psycho-cognitive performances as well as their associations in healthy, young and middle-aged MD versus PD workers. The data collection was undertaken by the same experienced exercise scientists and/or physiotherapists to avoid interobserver variations. Additionally, the tests used are established and reliable tools for assessing physical fitness and psychocognitive performances in the field (Frey et al. 1999; Burnstein et al. 2011; Suni et al. 2014; Tschopp et al. 2001; Latimer et al. 1999; Wolinsky et al. 2005; Hinton-Bayre and Geffen 2005;
Cohen et al. 1983; Silva Junior et al. 2013). Lastly, the magnitude of several fitness and psycho-cognitive outcomes such as handgrip strength, jump-and-reach performance, or perceived stress are similar to those previously observed in young and middleaged adults in Germany, Finland, and Denmark (Suni et al. 2014; Tittlbach et al. 2005; Jay et al. 2015).

We have to acknowledge a few limitations of this study. First, the present study used a cross-sectional study design. This design is the best way to determine prevalence and allows for assessing multiple outcomes (Mann 2003). Although cross-sectional studies are used to infer causation, they do not allow for cause-and-effect relations (Mann 2003). Second, factors such as lifestyle, socioeconomic status, and/or education could have affected physical fitness and/or psychocognitive performances. However, socioeconomic status is a latent construct relying on different indicators, thereby limiting data interpretation (Fliesser et al. 2018). Interestingly,

Table 3 Non-parametric rank correlation coefficient Spearman's Rho $(\rho)$ for the relationship between measures of physical fitness and psychocognitive performance

\begin{tabular}{|c|c|c|c|c|c|c|c|c|c|}
\hline & \multicolumn{3}{|c|}{ Cognitive performance } & \multicolumn{3}{|c|}{ Perceived stress } & \multicolumn{3}{|l|}{ Work ability } \\
\hline & MD group & PD group & $p$-Value & MD group & PD group & $p$-Value & MD group & PD group & $p$-Value \\
\hline $\mathrm{CRF}$ & 0.097 & -0.145 & $<0.10$ & 0.287 & 0.185 & n.s. & -0.237 & -0.219 & n.s. \\
\hline Handgrip strength & -0.205 & -0.075 & n.s. & -0.104 & -0.204 & n.s. & 0.004 & 0.149 & n.s. \\
\hline Trunk muscle endurance (flexors) & 0.125 & 0.134 & n.s. & -0.279 & -0.147 & n.s. & 0.301 & 0.071 & $<0.10$ \\
\hline Trunk muscle endurance (extensors) & 0.060 & 0.179 & n.s. & -0.199 & -0.123 & n.s. & 0.240 & -0.141 & $<0.05$ \\
\hline Jump performance & -0.065 & 0.258 & $<0.05$ & -0.259 & -0.033 & $<0.10$ & 0.040 & 0.055 & n.s. \\
\hline Static balance & 0.318 & 0.434 & n.s. & -0.128 & -0.059 & n.s. & 0.211 & -0.008 & n.s. \\
\hline
\end{tabular}

Statistically significant correlation coefficients are highlighted in bold. The $p$-value indicates whether the differences between groups were statistically significant

$M D$ workers with mental demands at work; $P D$ workers with physical demands at work 
studies indicated that different levels of socioeconomic status, education, and/or lifestyle are not or even adversely related with different fitness measures (Strand et al. 2011; Jensen et al. 2017; Koster et al. 2006). For instance, neither education nor occupation had an effect on grip strength in middle-aged men and women (Strand et al. 2011). Further, social class as operationalized by the level of education and job profile did not affect CRF (i.e., estimated $\mathrm{VO}_{2 \max }$ ) in a large sample of middle-aged, employed men (Jensen et al. 2017). Most importantly, effects of socioeconomic status as operationalized by the level of education and income on physical function in middle-aged men and women were predominantly explained by body mass index and LTPA (Koster et al. 2006). In the present study, body mass index as well as LTPA were assessed, thereby controlling indirectly for socioeconomic status. Nevertheless, future studies need to systematically include covariates such as socioeconomic status, education, or lifestyle. Third, we cannot rule out a selection bias during the recruitment process. In other words, workers with better performance measures could have been more likely to participate than workers with lower performance measures.

\section{Practical implications}

Recent review articles suggested to regularly include physical exercises into the daily routines at the workplace to counteract the negative side effects of occupational tasks (Sjøgaard et al. 2016; Søgaard and Sjøgaard 2017). In fact, given that most adults spend half of their waking hours at the workplace, the worksite setting offers a unique opportunity to promote physical activity and fitness. With respect to the present crosssectional findings, physical activity in the form of physical exercises should be increased to maintain or develop physical fitness and counteract the negative side effects of physical work demands. In particular, the correlation analysis indicated that gains in physical fitness may at least partly translate to benefits in psycho-cognitive performances and/or vice versa. Thus, physical exercises with the goal to improve physical fitness measures in the workforce could improve psychocognitive performances as well. Future studies should examine whether physical exercises can differently affect changes in physical fitness and psycho-cognitive performances in MD versus PD workers. These findings are essential before implementing work demand-specific public health promotion programs in the wider population.

\section{Conclusions}

Workers with primarily mental compared with physical work demands exhibit better balance, trunk extensor muscular endurance, and cognitive performance, as well as lower levels of perceived stress. Leisure-time physical activity (LTPA) modified the effects of physical work demands on physical fitness (i.e., trunk muscle endurance) and cognitive performance (i.e., digit symbol substitution test). Further, small-tomedium sized associations were found between physical fitness and psycho-cognitive performances, which indicates that gains in physical fitness may at least partly contribute to psycho-cognitive performance and/or vice versa, particularly in MD workers. Based on our findings, future longitudinal studies should examine the effects of physical versus mental demands at work on physical fitness and psycho-cognitive performance in young and middle-aged adults. Additionally, future research should look at cohort-specific (MD vs PD) effects of physical exercise programs on physical fitness and psycho-cognitive performances.

Acknowledgements We acknowledge the support of the health insurance company AOK Nordost, Germany, and the Potsdam Graduate School, Germany. Additionally, we would like to thank the participating companies and their employees, as well as Mr. Michael Herz, BA and Mr. Ron Borde, MA for their support during data collection.

Author contributions OP and UG: made substantial contributions to conception and design; OP and VL: contributed to data collection; OP, TD, and VL: carried out data analysis and interpretation together with $\mathrm{KG}$ and UG; OP: wrote the first draft of the manuscript and all authors were involved in revising it critically for important intellectual content; OP, TD, VL, KG, and UG: gave final approval of the version to be published and agreed to be accountable for all aspects of the work.

\section{Compliance with ethical standards}

Ethics approval and consent to participate Written informed consent was obtained from the participants before the start of the study. All experiments were approved by the ethics committee of the University of Potsdam (reference no. 29/2015) and conducted according to the latest version of the Declaration of Helsinki.

Conflict of interest The authors have no conflicts of interest that are directly relevant to the contents of this study.

Abbreviations CRF, Cardiorespiratory fitness; LTPA, Leisure-time physical activity; MD, Workers with mental demands at work; PD, Workers with physical demands at work; WAI, Work ability index

Open Access This article is distributed under the terms of the Creative Commons Attribution 4.0 International License (http:// creativecommons.org/licenses/by/4.0/), which permits unrestricted use, distribution, and reproduction in any medium, provided you give appropriate credit to the original author(s) and the source, provide a link to the Creative Commons license, and indicate if changes were made.

\section{References}

Biering-Sørensen F (1984) Physical measurements as risk indicators for low-back trouble over a one-year period. Spine 9(2):106-119

Bohannon RW, Larkin PA, Cook AC, Gear J, Singer J (1984) Decrease in timed balance test scores with aging. Phys Ther 64(7):1067-1070 
Bouchard C, Blair SN, Haskell WL (eds) (2012) Physical activity and health, 2nd edn. Human Kinetics, Champaign, IL

Bourban P, Hübner K, Tschopp M, Marti B (2001) Grundkraftanforderungen im Spitzensport: Ergebnisse eines 3teiligen Rumpfkrafttests. Schweiz Z Sportmed Sporttraumatol 49(2):73-78

Burnstein BD, Steele RJ, Shrier I (2011) Reliability of fitness tests using methods and time periods common in sport and occupational management. J Athl Train 46(5):505-513

Cassilhas RC, Viana VAR, Grassmann V, Santos RT, Santos RF, Tufik S, Mello MT (2007) The impact of resistance exercise on the cognitive function of the elderly. Med Sci Sports Exerc 39(8):1401-1407

Christensen JR, Kongstad MB, Sjøgaard G, Søgaard K (2015) Sickness presenteeism among health care workers and the effect of BMI, cardiorespiratory fitness, and muscle strength. J Occup Environ Med 57(12):e146-e152

Coenen P, Huysmans MA, Holtermann A, Krause N, van Mechelen W, Straker LM, van der Beek AJ (2018) Do highly physically active workers die early? A systematic review with meta-analysis of data from 193696 participants. Br J Sports Med 52:1320-1326

Cohen J (1992) A power primer. Psychol Bull 112(1):155-159

Cohen S, Kamarck T, Mermelstein R (1983) A global measure of perceived stress. J Health Soc Behav 24(4):385-396

Coren S (1993) The lateral preference inventory for measurement of handedness, footedness, eyedness, and earedness: norms for young adults. Bull Psychon Soc 31(1):1-3

Fairclough SH, Venables L, Tattersall A (2005) The influence of task demand and learning on the psychophysiological response. Int J Psychophysiol 56(2):171-184

Faul F, Erdfelder E, Buchner A, Lang A-G (2009) Statistical power analyses using $G^{*}$ Power 3.1: tests for correlation and regression analyses. Behav Res Methods 41(4):1149-1160

Fliesser M, de Witt Huberts J, Wippert P-M (2018) Education, job position, income or multidimensional indices? Associations between different socioeconomic status indicators and chronic low back pain in a German sample: a longitudinal field study. BMJ Open 8(4): e020207

Forcier K, Stroud LR, Papandonatos GD, Hitsman B, Reiches M, Krishnamoorthy J, Niaura R (2006) Links between physical fitness and cardiovascular reactivity and recovery to psychological stressors: a meta-analysis. Health Psychol 25(6):723-739

Frey I, Berg A, Grathwohl D, Keul J (1999) Freiburg questionnaire of physical activity - development, evaluation and application. Soz Praventivmed 44(2):55-64

García-Hermoso A, Cavero-Redondo I, Ramírez-Vélez R, Ruiz JR, Ortega FB, Lee D-C, Martínez-Vizcaíno V (2018) Muscular strength as a predictor of all-cause mortality in an apparently healthy population: a systematic review and meta-analysis of data from approximately 2 million men and women. Arch Phys Med Rehabil 99: 2100-2113.e5

Granacher U, Muehlbauer T, Gollhofer A, Kressig RW, Zahner L (2011a) An intergenerational approach in the promotion of balance and strength for fall prevention - a mini-review. Gerontol 57(4):304315

Granacher U, Wick C, Rueck N, Esposito C, Roth R, Zahner L (2011b) Promoting balance and strength in the middle-aged workforce. Int $\mathrm{J}$ Sports Med 32(1):35-44

Granacher U, Gollhofer A, Hortobágyi T, Kressig RW, Muehlbauer T (2013) The importance of trunk muscle strength for balance, functional performance, and fall prevention in seniors: a systematic review. Sports Med 43(7):627-641

Hinton-Bayre A, Geffen G (2005) Comparability, reliability, and practice effects on alternate forms of the Digit Symbol Substitution and Symbol Digit Modalities tests. Psychol Assess 17(2):237-241

Holtermann A, Mortensen OS, Burr H, Søgaard K, Gyntelberg F, Suadicani P (2010) Physical demands at work, physical fitness, and 30-year ischaemic heart disease and all-cause mortality in the Copenhagen male study. Scand J Work Environ Health 36(5):357365

Holtermann A, Hansen JV, Burr H, Søgaard K, Sjøgaard G (2012) The health paradox of occupational and leisure-time physical activity. $\mathrm{Br}$ J Sports Med 46(4):291-295

Ilmarinen J (2009) Work ability - a comprehensive concept for occupational health research and prevention. Scand J Work Environ Health 35(1): $1-5$

Jamnik VK, Gledhill N, Shephard RJ (2007) Revised clearance for participation in physical activity: greater screening responsibility for qualified university-educated fitness professionals. Appl Physiol Nutr Metab 32(6):1191-1197

Jay K, Friborg MK, Sjøgaard G, Jakobsen MD, Sundstrup E, Brandt M, Andersen LL (2015) The consequence of combined pain and stress on work ability in female laboratory technicians: a cross-sectional study. Int J Environ Res Public Health 12(12):15834-15842

Jensen MT, Holtermann A, Bay H, Gyntelberg F (2017) Cardiorespiratory fitness and death from cancer: a 42-year followup from the Copenhagen Male Study. Br J Sports Med 51(18):13641369

Koster A, Bosma H, Broese van Groenou MI, Kempen GIJM, Penninx BWJH, van Eijk JTM, Deeg DJH (2006) Explanations of socioeconomic differences in changes in physical function in older adults: results from the Longitudinal Aging Study Amsterdam. BMC Public Health 6:244

Latimer J, Maher CG, Refshauge K, Colaco I (1999) The reliability and validity of the Biering-Sorensen test in asymptomatic subjects and subjects reporting current or previous nonspecific low back pain. Spine 24(20):2085-2089

Lezak MD, Howieson DB, Bigler ED, Tranel D (2012) Neuropsychological assessment. Oxford University Press, Oxford

Liu-Ambrose T, Nagamatsu LS, Graf P, Beattie BL, Ashe MC, Handy TC (2010) Resistance training and executive functions: a 12-month randomized controlled trial. Arch Intern Med 170(2):170-178

Mann CJ (2003) Observational research methods. Research design II: cohort, cross sectional, and case-control studies. Emerg Med J 20(1):54-60

Moore JB, Beets MW, Barr-Anderson DJ, Evenson KR (2013) Sedentary time and vigorous physical activity are independently associated with cardiorespiratory fitness in middle school youth. J Sports Sci 31(14):1520-1525

Myrtek M, Fichtler A, Strittmatter M, Brügner G (1999) Stress and strain of blue and white collar workers during work and leisure time: results of psychophysiological and behavioral monitoring. Appl Ergon 30(4):341-351

Newson RS, Kemps EB (2008) Relationship between fitness and cognitive performance in younger and older adults. Psychol Health 23(3): 369-386

Nsenga Leunkeu A, Shephard RJ, Ahmaidi S (2014) A brief history of exercise clearance and prescription: 1 . The era of heart rate recovery curves. Health Fitness J Can 7(1):26-35

Ryhming I (1953) A modified Harvard step test for the evaluation of physical fitness. Arbeitsphysiologie 15(3):235-250

Savinainen M, Nygård C-H, Ilmarinen J (2004) A 16-year follow-up study of physical capacity in relation to perceived workload among ageing employees. Ergonomics 47(10):1087-1102

Silva Junior SHA, Vasconcelos AGG, Griep RH, Rotenberg L (2013) Test-retest reliability of the Work Ability Index (WAI) in nursing workers. Rev Bras Epidemiol 16(1):202-209

Sjøgaard G, Christensen JR, Justesen JB, Murray M, Dalager T, Fredslund GH, Søgaard K (2016) Exercise is more than medicine: the working age population's well-being and productivity. J Sport Health Sci 5(2):159-165 
Søgaard K, Sjøgaard G (2017) Physical activity as cause and cure of muscular pain: evidence of underlying mechanisms. Exerc Sport Sci Rev 45(3):136-145

Strand BH, Cooper R, Hardy R, Kuh D, Guralnik J (2011) Lifelong socioeconomic position and physical performance in midlife: results from the British 1946 birth cohort. Eur J Epidemiol 26(6):475-483

Suni JH, Rinne MB, Ruiz JR (2014) Retest repeatability of motor and musculoskeletal fitness tests for public health monitoring of adult populations. J Nov Physiother 4(1):1000194

Teisala T, Mutikainen S, Tolvanen A, Rottensteiner M, Leskinen T, Kaprio J, Kolehmainen M, Rusko H, Kujala UM (2014) Associations of physical activity, fitness, and body composition with heart rate variability-based indicators of stress and recovery on workdays: a cross-sectional study. J Occup Med Toxicol 9:16

Then FS, Luck T, Luppa M, Arélin K, Schroeter ML, Engel C, Löffler M, Thiery J, Villringer A, Riedel-Heller SG (2014) Association between mental demands at work and cognitive functioning in the general population - results of the health study of the Leipzig research center for civilization diseases (LIFE). J Occup Med Toxicol 9:23

Tittlbach S, Kolb H, Woll A, Bös K (2005) Health related fitness test battery (KGFT). B \& G 21(3):109-115
Tonello L, Rodrigues FB, Souza JWS, Campbell CSG, Leicht AS, Boullosa DA (2014) The role of physical activity and heart rate variability for the control of work related stress. Front Physiol 5:67

Torgén M, Punnett L, Alfredsson L, Kilbom A (1999) Physical capacity in relation to present and past physical load at work: a study of 484 men and women aged 41 to 58 years. Am J Ind Med 36(3):388-400

Tschopp M, Bourban P, Hübner K, Marti B (2001) Messgenauigkeit eines 4-teiligen, standardisierten dynamischen Rumpfkrafttests: Erfahrungen mit gesunden männlichen Spitzensportlern. Schweiz Z Sportmed Sporttraumatol 49(2):67-72

Wolinsky FD, Miller DK, Andresen EM, Malmstrom TK, Miller JP (2005) Reproducibility of physical performance and physiologic assessments. J Aging Health 17(2):111-124

Wu B, Porell F (2000) Job characteristics and leisure physical activity. J Aging Health 12(4):538-559

Publisher's note Springer Nature remains neutral with regard to jurisdictional claims in published maps and institutional affiliations. 